\section{THE SIGNIFICANCE OF ACUTE-PHASE SMALL-FOR-SIZE LIVER GRAFT INJURY IN MOBILIZATION OF CIRCULATING EPCS/ MDSCS/TREGS AFTER LDLT FOR HCC PATIENTS}

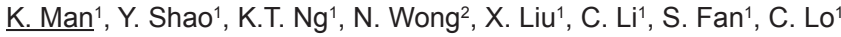 \\ ${ }^{1}$ Department Of Surgery, The University of Hong Kong, Hong Kong/ \\ HONG KONG, ${ }^{2}$ Antatomical And Cellular Pathology, Chinese \\ University of Hong Kong, Hong Kong/HONG KONG
}

Introduction and Objective: Higher incidence of tumor recurrence is a major obstacle of living donor liver transplanatation (LDLT) for the patients with hepatocellular carcinoma (HCC). We have already demonstrated that acute phase small-for-size liver graft injury plays important role on late phase tumor recurrence and metastases in a serial animal studies. Understanding the molecular mechanism of acute phase small-for-size liver graft injury is essential for development of therapeutic strategy to reduce the likelihood of tumor recurrence after LDLT. In the current clinical study, we aim to investigate the impact of acute-phase small-for-size graft injury on mobilization of circulating endothelial progenitor cells (EPCs), myeloid-derived suppressive cells (MDSCs) and regulatory T cells (Tregs) in HCC patients after liver transplantation and to explore the molecular mechanism therein.

Methods: From May 2000 to November 2009, 115 adult HCC recipients were included in the current study. The intragraft microRNA profiles of the grafts greater (Group 1) and less than 60\% (Group 2) of standard liver weight (SLW) were characterized by Low Density Array (LDA) analysis. Post-operative circulating EPCs (CD34+CD133+CD45-), MDSCs (CD34+CD13+CD33+) and Tregs (CD4+CD25+FOXP3+) were compared by FACS analysis. Intragraft hepatic stellate cell activation, macrophage infiltration and gene expression of Rac, Pyk2, Egr-1 and VEGF at the early phase after reperfusion were also detected by immunostaining and real-time RT$\mathrm{PCR}$, respectively. Clinical-pathological data including the incidence of tumor recurrence and metastasis were compared between the two groups. Results: The patients were grouped into Group 1 (>= $60 \%$ SLW, $n=37)$ and Group $2(<60 \%$ SLW, $n=78)$. The numbers of patients beyond Milan criteria [15/37(40.5\%) vs 29/49(59.2\%), $p=0.838]$ or UCSF criteria [9/37(24.3\%) vs $19 / 60(31.7 \%), p=1]$ were similar between the two groups. Much more patients in Group 2 developed tumor recurrence and lung metastasis $[19 / 78(24.4 \%)$ vs $3 / 37(8 \%)$, $\mathrm{p}=0.04]$. Level of circulating EPCs was significantly higher in Group 2 (Day 3: $0.09 \%$ vs $0.002 \%, p=0.019$; Week $4: 0.12 \%$ vs $0.033 \%$, $p=0.037$; Week 8 : $0.0585 \%$ vs $0.025 \%, p=0.018$; Week $12: 0.055 \%$ vs $0.028 \%, p=0.025$ ). A tendency of larger populations of circulating MDSCs and Tregs was also found in Group 2. Most of the patients with tumor recurrence had hepatic sinusoidal injury at early phase after liver transplantation. Significant activation of hepatic stellate cells was found in Group 2 together with stronger intragraft protein expression of FAK and CAK compared to Group 1. Intragraft mRNA levels of Egr-1, RhoA, FAK and VEGF were also significantly higher in Group 2. microRNA LDA analysis demonstrated that mir-233, mir-141, mir-1308, mir-548 and mir-576 were differentially expressed between the two groups. These mirRNAs were predicted to regulate targeting genes linked to graft injury (MAPK, CCL4 and Egr-1), tumor invasiveness (STAT5, CDC2 and EGFR), angiogenesis (VEGF, FLT4 and ANGPTL5), and macrophage infiltration (MIP2). Conclusion: A significantly higher population of postoperative circulating EPCs, which are mobilized by small-for-size graft injury, may lead to a higher incidence of tumor recurrence and metastasis after LDLT. The distinct intragraft miRNA expression profile linked to acute-phase injury and angiogenesis may play a role in the mobilization of circulating EPCs, MDSCs, and Tregs.

Disclosure: All authors have declared no conflicts of interest.

\section{DUAL LIVING-DONOR LIVER TRANSPLANTATION AT THE SINGLE CENTER}

S. Lee $^{1}$, S. Hwang' ${ }^{1}$ K. Kim¹, C. Ahn², D. Moon², T. Ha², G. Song ${ }^{1}$, J. Dong-Hwan', S. Sim¹, S. Kim ${ }^{1}$

${ }^{1}$ Division Of Liver Transplantation And Hb Surgery, Department Of Surgery, Asan Medical Center, Seoul/KOREA, ${ }^{2}$ Division Of Hepatobiliary Surgery And Liver Transplantation, Department Of Surgery, Asan Medical Center, University of Ulsan College of Medicine, Seoul/KOREA

(Introduction)The major limitation of adult-to-adult living donor liver transplantation (AALDLT) is the adequacy of graft size and the issue of donor safety. It has been known that near $50 \%$ of the standard liver volume of the recipient is the minimally required graft volume to provide the adequate functional liver mass. A left lobe from the relatively small donor will not meet the metabolic demands of the larger recipient. The possible solution to this problem is to increase the extent of the donor's liver resection by harvesting the right lobe, which accounts for more than $60 \%$ to $70 \%$ of total liver volume. However, the right lobe hepatectomy in the donor is not always safe, depending on the volume of the remaining left lobe. Not all donor can provide their right lobe because the safe donation is possible only when the future remnant liver volume is over $30 \%$. As an alternative, dual left lobe from two living donors can solve the problem of graftsize insufficiency and guarantee donor safety. In many occasions, the recipient's body size is big, but the potential donors' body size is small and their right lobe alone cannot meet the metabolic demand of the recipient. Dual transplantation with a right lobe and a left lobe will suffice the requirement of GRWR for this particular patient. (Method)From 2000 March to 2009 December, 291 Dual LDLTs were performed at our institute with 221 two left-lobes, 56 right + left lobes, and 14 posterior sector + left lobe. There were 62 emergent or urgent cases for fulminant and acute-on-chronic liver failure. (Results) In-hospital mortality occurred in $20(6.8 \%)$ of 291 cases. Indications and survival outcomes of dual LDLT are comparable to those of single right-lobe LDLT. Although it seems to be complexed operation, it allows for provision of adequate graft mass for the recipients who would otherwise have no chance if only one donor were considered. By adding dual transplantation program, at least $12 \%$ increase of adult LDLT numbers can be anticipated.(Conclusion) The dual LDLT can provide sufficient graft volume without increasing donor risk.

Disclosure: All authors have declared no conflicts of interest. 